\title{
Accelerated large volume irradiation with dynamic Jaw/Dynamic Couch Helical Tomotherapy
}

\author{
Sonja Krause ${ }^{1 *}$, Sebastian Beck ${ }^{1}$, Kai Schubert ${ }^{1}$, Steffen Lissner ${ }^{1}$, Susanta Hui ${ }^{2}$, Klaus Herfarth', Juergen Debus ${ }^{1}$ \\ and Florian Sterzing ${ }^{1}$
}

\begin{abstract}
Background: Helical Tomotherapy $(\mathrm{HT})$ has unique capacities for the radiotherapy of large and complicated target volumes. Next generation Dynamic Jaw/Dynamic Couch HT delivery promises faster treatments and reduced exposure of organs at risk due to a reduced dose penumbra.

Methods: Three challenging clinical situations were chosen for comparison between Regular HT delivery with a field width of $2.5 \mathrm{~cm}$ (Reg 2.5) and $5.0 \mathrm{~cm}$ (Reg 5.0) and DJDC delivery with a maximum field width of $5.0 \mathrm{~cm}$ (DJDC 5.0): Hemithoracic Irradiation, Whole Abdominal Irradiation (WAI) and Total Marrow Irradiation (TMI). For each setting, five $C T$ data sets were chosen, and target coverage, conformity, integral dose, dose exposure of organs at risk (OAR) and treatment time were calculated.

Results: Both Reg 5.0 and DJDC 5.0 achieved a substantial reduction in treatment time while maintaining similar dose coverage. Treatment time could be reduced from 10:57 $\mathrm{min}$ to 3:42 $\mathrm{min} /$ 5:10 $\mathrm{min}$ (Reg 5.0 / DJDC 5.0) for Hemithoracic Irradiation, from 18:03 min to 8:02 $\mathrm{min}$ / 8:03 min for WAl and to 18:25 min / 18:03 min for TMI. In Hemithoracic Irradiation, OAR exposure was identical in all modalities. For WAI, Reg 2.5 resulted in lower exposure of liver and bone. DJDC plans showed a small but significant increase of $\sim 1$ Gy to the kidneys, the parotid glans and the thyroid gland. While Reg 5.0 and DJDC were identical in terms of OAR exposure, integral dose was substantially lower with DJDC, caused by a smaller dose penumbra.
\end{abstract}

Conclusions: Although not clinically available yet, next generation DJDC HT technique is efficient in improving the treatment time while maintaining comparable plan quality.

Keywords: Dynamic jaw/dynamic couch, Helical tomotherapy, Large volumes, Hemithoracic irradiation, Whole abdominal irradiation, Total marrow irradiation

\section{Background}

Helical Tomotherapy (HT) has proven to have unique capacities for the radiotherapy treatment of large target volumes [1,2]. Technical details of HT have been described extensively before [3]. In brief, a HT unit with its ring gantry forms a fusion of a linear accelerator and a helical CT scanner [4]. The treatment beam can be shaped into different beam widths depending on the opening angle of the secondary collimator (jaws). In an

\footnotetext{
* Correspondence: sonja.krause@med.uni-heidelberg.de

${ }^{1}$ Department of Radiation Oncology, University Hospital Heidelberg, INF 400, 69120, Heidelberg, Germany

Full list of author information is available at the end of the article
}

inverse treatment planning process, the MLC conformation is optimised to obtain highly conformal radiation doses to the target [5]. As the patient is moved through the gantry, targets up to a length of $160 \mathrm{~cm}$ can be treated in one treatment run. While HT can yield excellent target coverage and sparing of organs at risk in the treatment of very large volumes [6], it has often been criticised because of long treatment times.

During the last years, other techniques of rotational IMRT have evolved. As initially described by Otto [7], in volumetric modulated arc therapy dose is delivered by a modulated beam with a field size of up to $40 \times 40 \mathrm{~cm}$ using one or very few rotations. This technique offers

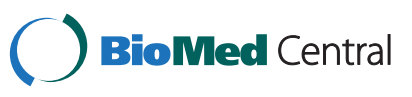


very fast IMRT treatments, especially for target volumes of low or intermediate complexity [8]. Satisfactory coverage of complex volumes frequently needs more than one rotation, and very large target extensions often demand multiple isocenters that require additional patient setup time.

With HT, the problem of field junctioning in large targets has been solved by the helical superposition of multiple beam rotations. However, treatment time is still a crucial factor influencing treatment safety, as patient motion and the subsequent risk of dose uncertainties increases with treatment time. New HT developments such as Dynamic Jaw/Dynamic Couch (DJDC) have the potential to speed up treatment substantially: While in current HT the field width (determined by the jaw opening) as well as the couch speed remain constant once chosen at the beginning of treatment planning, DJDC allows for the dynamic adaptation of field width and couch speed depending on target complexity. Thus, areas with less complex target shapes can be treated fast and with a broad field width without compromising OAR sparing and target coverage in more critical localisations $[9,10]$. In addition, dynamic opening of the jaws at the start and end of the target reduces the craniocaudal dose penumbra caused by a constant field width. Currently, DJDC is still in development and not available for clinical routine yet.

To put DJDC to the test, we chose three clinical settings where the radiation oncologist is faced with the challenge of treating very large and complex volumes in close proximity to critical organs at risk. The first was the treatment of one complete hemithorax after radical surgery for Malignant Pleural Mesothelioma. Secondly, we investigated Whole Abdominal Irradiation (WAI) after surgery for ovarian cancer, and thirdly the treatment of the complete bone marrow (Total Marrow Irradiation, TMI) as part of conditioning regimens before stem cell transplantation.

The present planning study compares state-of-the-art HT with $2.5 \mathrm{~cm}$ and $5.0 \mathrm{~cm}$ field width to DJDC and characterises the benefits of future HT developments for the treatment of three very large volumes: Hemithoracic Irradiation, WAI and TMI.

\section{Materials and methods}

This plan comparison study consisted of 15 anonymised clinical cases forming three groups: 5 patients with Malignant Pleural Mesothelioma had obtained a hemithoracic irradiation with HT and 5 patients had been treated with a HT Whole Abdominal Irradiation (WAI) for ovarian cancer FIGO IIIc. In preparation for a clinical Total Marrow Irradiation (TMI) trial, the plans for TMI were conducted on 5 planning CT scans of patients that had obtained a craniospinal irradiation with a suitable CT scan length. For all settings, the original CT scans, and for hemithoracic irradiation and WAI also the original structure sets were used to generate Reg 2.5, Reg 5 and DJDC plans on the original data sets. All plans were calculated and evaluated by the same two persons to keep variations in planning procedure als low as possible.

\section{Hemithoracic irradiation}

Two patients suffered from left-sided, three patients from right-sided mesothelioma. All 5 patients had obtained neoadjuvant chemotherapy with cisplatin and pemetrexed and extrapleural pneumonectomy and had been treated with regular HT Hemithoracic irradiation using a field width of $2.5 \mathrm{~cm}$ (Reg 2.5), because in clinical routine, a $5.0 \mathrm{~cm}$ field width resulted in larger exposure of the neck as well as the left kidney or the liver. Planning CT scans with $3.0 \mathrm{~mm}$ slices were used. The PTV included the former pleural cavity and the surgical scars with a security margin of $1.0 \mathrm{~cm}$ and had an average volume of $3199 \mathrm{ml}$. A median dose of $54.0 \mathrm{~Gy}$ in 27 fractions was prescribed to the PTV. Reg 2.5 plans were compared to Reg 5.0 and DJDC 5.0 plans in terms of treatment time, target coverage and exposure of organs at risk (OAR). All HT modalities were planned on a fine calculation grid with a pitch of 0.287 . For Reg 2.5 and Reg 5.0, the Intensity Modulation Factor (IMF; maximum leaf intensity divided by the average leaf intensity) was increased gradually up to 2.4, while for DJDC an IMF of 2.2-2.5 was reached. Of note, the IMF for DJDC represents the value given during planning, not the actual IMF calculated by the planning system, as the research version does not allow the specification of a commissioned treatment machine and consecutively does not permit a final dose calculation.

\section{Whole abdominal irradiation}

All 5 patients were treated for ovarian cancer stage FIGO IIIc after resection of the ovaries and the peritoneum including all macroscopic tumor lesions and adjuvant platinum-based chemotherapy. The PTV consisted of the complete peritoneal cavity with a security margin of $1.5 \mathrm{~cm}$ (2.5 cm in craniocaudal direction). For HT planning purposes, the PTV was subdivided in order to control underdosage due to OAR sparing: Two PTVs surrounded the left and right kidneys, one covered the upper part of the abdomen close to the lung and the fourth PTV covered the rest of the abdominal cavity. Planning CT scans of $5.0 \mathrm{~mm}$ slice thickness were used. A median dose of 30.0 Gy in 1.5 Gy fractions was prescribed. Again, the Reg 2.5 plans used in clinical routine were compared to Reg 5.0 and DJDC 5.0 plans. Both HT plans had a pitch of 0.287 ; Reg 2.5 and Reg 5.0 plans needed an IMF of 4.0 while DJDC planning resulted in an IMF of $3.6-4.0$. 


\section{Total marrow irradiation}

In preparation for clinical phase I TMI trial, planning CT scans with $5 \mathrm{~mm}$ slice thickness of patients receiving craniospinal irradiation were chosen for TMI planning because of the suitable scan length. The CTV comprised the complete bone marrow with the exception of the mandible and maxillary bones for a better sparing of the oral mucosa. For PTV construction, different security margins were applied depending on localisation: For the skull down to $\mathrm{C} 2$, no security margin was applied as the fixation with a rigid mask ensures precise positioning, on other locations, a security margin of $5 \mathrm{~mm}$ was chosen. A median of 18 Gy in 9 fractions was prescribed to the PTV. As for the other indications, Reg 2.5 was compared to Reg 5.0 and DJDC 5.0. To account for the large craniocaudal extension of the PTV, a pitch of 0.43 was chosen for HT plans; the IMF was increased up to 2.5 for all HT plans.

\section{Plan comparison and statistical analysis}

Plan quality concerning the PTV was judged using $\mathrm{D}_{99}$ and $\mathrm{D}_{1}$ (dose to $99 \%$ and $1 \%$ of the target volume), $\mathrm{CI}_{95}$ (Conformity Index; total volume covered by the $95 \%$ isodose divided by the volume of the PTV covered by the 95\% isodose), UI (Uniformity Index; dose covering 5\% of the PTV divided by the dose covering 95\% of the PTV) and $\mathrm{TV}_{95 \%}$ (PTV volume covered by $95 \%$ of the prescribed dose divided by the PTV volume). Sparing of organs at risk was judged by maximum and average dose as well as integral dose (product of the total volume and the mean dose to that volume).
For statistical analysis, a two-sided paired t-test was used. A value of $\mathrm{p}<0.05$ was considered statistically significant.

\section{Results}

\section{Treatment time}

Regular HT with $2.5 \mathrm{~cm}$ field width of all three types of complex and very large target volumes took an average of 10:57 $\mathrm{min}$ (Hemithoracic Irradiation), 18:03 $\mathrm{min}$ (WAI) and 39:23 min (TMI). Using a field width of $5.0 \mathrm{~cm}$ in regular delivery more than halved average treatment time to 3:42 min for Hemithoracic Irradiation, 8:02 min for WAI and 18:25 min for TMI. With DJDC technique, Hemithoracic Irradiation took longer than with Reg 5.0 (5:01 min, not significant), while WAI and TMI treatments were as fast as with Reg 5.0 (8:03 $\mathrm{min}$ and 19:01 $\mathrm{min}$ ) (see Figure 1). While treatment speed was comparable for DJDC and Reg 5.0, the latter produced a larger craniocaudal dose penumbra and consecutively a higher integral dose (see below).

\section{Target coverage}

Although for DJDC planning a maximum field width twice as large as in regular delivery was permitted, dose distribution in the target volumes remained on a similar level (see Tables 1, 2, 3 and Figures 2, 3, 4). Target coverage as indicated by TV95\% was almost identical in all modalities for Hemithoracic Irradiation and TMI. For WAI, a significantly better coverage could be attained with Reg 2.5.

Target coverage in the chest wall dropped in all modalities in proximity to the lungs to a level of about 12

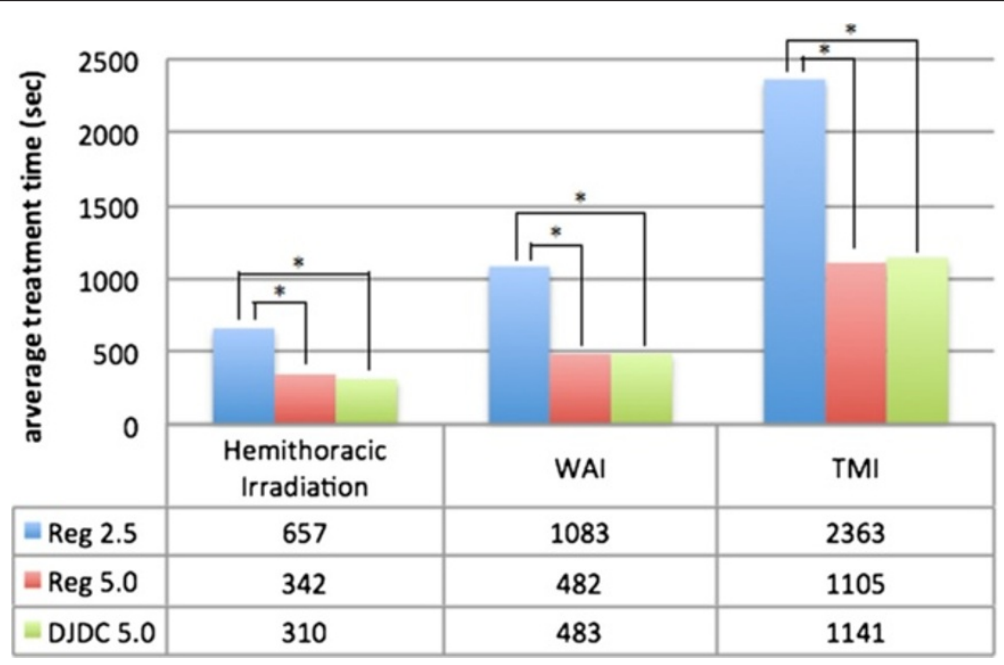

Figure 1 Average treatment times (sec) for Hemithoracic Irradiation, Whole Abdominal Irradiation (WAI) and Total Marrow Irradiation (TMI) for the Reg. 2.5, Reg 5.0 ("regular" delivery with a $2.5 \mathrm{~cm}$ and 5.0 field width) and DJDC 5.0 (Dynamic Jaw/Dynamic Couch delivery with a $5.0 \mathrm{~cm}$ maximum field width) plans. In all three plan groups, DJDC 5.0 came up with significantly shorter treatment times compared to Reg 2.5 ( $\left.^{*} p<005\right)$. 
Table 1 Hemithoracic irradiation: plan characteristics for Reg 2.5, Reg 5.0 ("regular" delivery with a $2.5 \mathrm{~cm}$ and 5.0 cm field width) and DJDC 5.0 (dynamic jaw/dynamic couch delivery with a $5.0 \mathrm{~cm}$ maximum field width)

\begin{tabular}{llccc}
\hline & & Reg 2.5 & Reg 5.0 & DJDC 5.0 \\
\hline PTV & $\mathrm{D}_{99}$ (Gy) & $48.6 \pm 0.6$ & $49.5 \pm 1.0$ & $48.7 \pm 0.6$ \\
\hline & $\mathrm{D}_{1}$ (Gy) & $56.0 \pm 0.5$ & $57.7 \pm 1.3$ & $55.9 \pm 0.2$ \\
\hline & $\mathrm{Cl}_{95}$ & $1.14 \pm 0.08$ & $1.19 \pm 0.06$ & $1.16 \pm 0.05$ \\
\hline & $\mathrm{UI}$ & $1.06 \pm 0.03$ & $1.09 \pm 0.04$ & $1.06 \pm 0.00$ \\
\hline & $\mathrm{TV}_{95 \%}$ & $0.967 \pm 0.015$ & $0.975 \pm 0.009$ & $0.967 \pm 0.004$ \\
\hline liver & mean (Gy) & $20.1 \pm 9.1$ & $20.7 \pm 9.4$ & $19.9 \pm 10.1$ \\
\hline $\begin{array}{l}\text { ipsilateral } \\
\text { kidney }\end{array}$ & mean (Gy) & $8.8 \pm 3.0$ & $8.3 \pm 2.9$ & $8.4 \pm 2.6$ \\
\hline $\begin{array}{l}\text { contralateral } \\
\text { kidney }\end{array}$ & mean (Gy) & $4.0 \pm 1.2$ & $3.6 \pm 0.9$ & $3.3 \pm 1.2$ \\
\hline $\begin{array}{l}\text { contralateral } \\
\text { lung }\end{array}$ & mean (Gy) & $6.1 \pm 0.6$ & $6.2 \pm 0.7$ & $6.0 \pm 1.0$ \\
\hline spinal cord & max (Gy) & $35.0 \pm 6.2$ & $32.3 \pm 0.9$ & $34.1 \pm 2.1$ \\
\hline heart & mean (Gy) & $26.5 \pm 4.8$ & $26.9 \pm 6.9$ & $26.7 \pm 5.3$ \\
\hline esophagus & mean (Gy) & $32.0 \pm 3.2$ & $32.5 \pm 1.2$ & $31.5 \pm 3.1$ \\
\hline integral dose & (Gy x I) & $411.4 \pm 24.5$ & $513 \pm 110.9$ & $414.6 \pm 31.3$ \\
\hline
\end{tabular}

No statistically significant differences could be detected in dose distribution in the PTV and sparing of organs at risk.

Gy. This effect was more pronounced when a $5 \mathrm{~cm}$ field width was used. In Figure 4, the equivalent lung sparing and dose fall-off in the target is indicated by light arrowheads.

Conformity was on the same level in all delivery modes for Hemithoracic Irradiation and WAI. In the TMI plans, having the largest and most complex target volumes, conformity was slightly, yet significantly, worse for Reg 5.0 and DJDC 5.0 as compared to Reg 2.5 (see Table 3).

Table 2 Whole abdominal irradiation: plan characteristics for Reg 2.5, Reg 5.0 ("regular" delivery with a $2.5 \mathrm{~cm}$ and $5.0 \mathrm{~cm}$ field width) and DJDC 5.0 (dynamic jaw/dynamic couch delivery with a $5.0 \mathrm{~cm}$ maximum field width)

\begin{tabular}{llccc}
\hline & & Reg $\mathbf{2 . 5}$ & Reg $\mathbf{5 . 0}$ & DJDC $\mathbf{5 . 0}$ \\
\hline total PTV & $\mathrm{Cl}_{95}$ & $1.11 \pm 0.08$ & $1.13 \pm 0.04$ & $1.16 \pm 0.03$ \\
\hline & $\mathrm{TV}_{95 \%}$ & $\mathbf{0 . 9 2 5} \pm \mathbf{0 . 0 1 3}$ & $\mathbf{0 . 8 8 7} \pm \mathbf{0 . 0 0 7}$ & $\mathbf{0 . 9 3 1} \pm \mathbf{0 . 0 2 2}$ \\
\hline $\begin{array}{l}\text { right } \\
\text { kidney }\end{array}$ & mean (Gy) & $11.9 \pm 0.8$ & $12.3 \pm 1.1$ & $13.0 \pm 0.9$ \\
\hline left kidney & mean (Gy) & $10.7 \pm 0.8$ & $11.7 \pm 1.0$ & $11.5 \pm 0.8$ \\
\hline bone & mean (Gy) & $\mathbf{1 0 . 8} \pm \mathbf{1 . 1}$ & $\mathbf{1 2 . 9} \pm \mathbf{1 . 0}$ & $11.7 \pm 0.8$ \\
\hline spinal cord & max (Gy) & $12.7 \pm 2.7$ & $12.5 \pm 1.1$ & $13.6 \pm 2.7$ \\
\hline heart & mean (Gy) & $9.5 \pm 2.4$ & $10.6 \pm 2.7$ & $10.5 \pm 1.5$ \\
\hline liver & mean (Gy) & $\mathbf{2 2 . 3} \pm \mathbf{0 . 9}$ & $\mathbf{2 3 . 5} \pm \mathbf{0 . 5}$ & $\mathbf{2 3 . 7} \pm \mathbf{0 . 9}$ \\
\hline $\begin{array}{l}\text { integral } \\
\text { dose }\end{array}$ & $(\mathrm{Gy} \times \mathrm{l})$ & $\mathbf{3 6 9 . 3} \pm \mathbf{6 7 . 8}$ & $\mathbf{4 9 6 . 2} \pm \mathbf{9 2 . 3}$ & $\mathbf{3 8 2 . 9} \pm \mathbf{5 2 . 4}$
\end{tabular}

Target coverage and bone and liver sparing were significantly better with Reg 2.5 (bold print). Reg 5.0 resulted in a significantly higher integral dose than Reg 2.5 and DJDC 5.0 (bold print).
Table 3 Total marrow irradiation: plan characteristics for Reg 2.5, Reg 5.0 ("regular" delivery with a $2.5 \mathrm{~cm}$ and 5.0 cm field width) and DJDC 5.0 (dynamic jaw/dynamic couch delivery with a $5.0 \mathrm{~cm}$ maximum field width)

\begin{tabular}{llccc}
\hline & & Reg $\mathbf{2 . 5}$ & Reg 5.0 & DJDC 5.0 \\
\hline PTV & D99 (Gy) & $12.2 \pm 0.6$ & $11.9 \pm 0.6$ & $11.9 \pm 0.3$ \\
& D1 (Gy) & $19.6 \pm 0.3$ & $20.1 \pm 0.4$ & $19.6 \pm 0.3$ \\
& Cl$_{\mathbf{9 5}}$ & $\mathbf{1 . 3 0} \pm \mathbf{0 . 1 5}$ & $\mathbf{1 . 7} \pm \mathbf{0 . 2 3}$ & $\mathbf{1 . 4 7} \pm \mathbf{0 . 0 5}$ \\
& UI & $1.27 \pm 0.05$ & $1.34 \pm 0.08$ & $1.31 \pm 0.07$ \\
& TV95\% & $0.781 \pm 0.074$ & $0.768 \pm 0.037$ & $0.768 \pm 0.057$ \\
bladder & mean (Gy) & $6.8 \pm 1.3$ & $8.2 \pm 1.1$ & $8.0 \pm 1.8$ \\
small bowel & mean (Gy) & $6.8 \pm 1.2$ & $8.2 \pm 1.1$ & $7.2 \pm 1.0$ \\
& D50 (Gy) & $6.0 \pm 0.9$ & $8.1 \pm 3.7$ & $6.6 \pm 1.0$ \\
esophagus & mean (Gy) & $6.7 \pm 1.2$ & $9.0 \pm 0.5$ & $7.2 \pm 1.0$ \\
brain & mean (Gy) & $8.3 \pm 0.6$ & $8.1 \pm 1.3$ & $8.7 \pm 1.6$ \\
& D50 (Gy) & $6.9 \pm 0.8$ & $7.2 \pm 1.7$ & $8.1 \pm 0.3$ \\
liver & mean (Gy) & $\mathbf{7 . 6} \pm \mathbf{0 . 4}$ & $8.3 \pm 0.5$ & $\mathbf{8 . 5} \pm \mathbf{0 . 6}$ \\
lenses & mean (Gy) & $2.4 \pm 0.2$ & $2.5 \pm 0.3$ & $2.7 \pm 0.3$ \\
right lung & mean (Gy) & $8.0 \pm 0.2$ & $8.2 \pm 0.3$ & $8.1 \pm 0.1$ \\
& D50 (Gy) & $\mathbf{6 . 2} \pm \mathbf{0 . 3}$ & $6.7 \pm 0.3$ & $\mathbf{6 . 6} \pm \mathbf{0 . 1}$ \\
left lung & mean (Gy) & $8.0 \pm 0.1$ & $8.1 \pm 0.3$ & $8.1 \pm 0.3$ \\
& D50 (Gy) & $\mathbf{6 . 3} \pm \mathbf{0 . 1}$ & $6.7 \pm 0.3$ & $\mathbf{6 . 6} \pm \mathbf{0 . 1}$ \\
stomach & mean (Gy) & $5.2 \pm 0.8$ & $6.6 \pm 1.1$ & $5.9 \pm 0.7$ \\
kidneys & mean (Gy) & $\mathbf{6 . 1} \pm \mathbf{0 . 6}$ & $6.0 \pm 0.8$ & $\mathbf{7 . 1} \pm \mathbf{0 . 8}$ \\
parotid glands & mean (Gy) & $\mathbf{4 . 2} \pm \mathbf{0 . 8}$ & $4.2 \pm 0.9$ & $\mathbf{5 . 8} \pm \mathbf{1 . 2}$ \\
thyroid gland & mean (Gy) & $\mathbf{4 . 2} \pm \mathbf{0 . 5}$ & $4.9 \pm 0.5$ & $\mathbf{5 . 1} \pm \mathbf{0 . 5}$
\end{tabular}

integral dose $\quad(G y \times I) \quad 631.9 \pm 143.4 \quad 724.5 \pm 140.6 \quad 690.0 \pm 122.3$

Conformity was superior in Reg 2.5 compared to Reg 2.5 and DJDC 5.0 (bold print). Reg 2.5 slightly outperformed DJDC 5.0 in terms of liver, kidney, parotid gland and thyroid sparing and D50 to both lungs (bold print).

\section{OAR sparing and integral dose}

With the dynamic opening and closing of the secondary collimator at the start and end of the target in DJDC 5.0 plans, a smaller craniocaudal dose penumbra compared to Reg 2.5 and Reg 5.0 plans could be realised (see Figures 5 and 6), allowing a better genital sparing in WAI and a sparing of the neck and supraclavicular area in Hemithoracic Irradiation. Consecutively, integral dose was significantly lower in WAI plans when using DJDC 5.0 instead of Reg 2.5 or Reg 5.0. In Hemithoracic Irradiation and TMI, the reduction of integral dose by DJDC compared to Reg 5.0 did not reach statistical significance. In all settings, DJDC 5.0 resulted in a slightly higher integral dose than Reg 2.5 (not significant).

Consequentially, DJDC plans showed a tendency to a higher exposure of organs at risk. In Hemithoracic Irradiation, no significant increase could be detected, with the cardinal organ at risk, the contralateral lung, receiving 6.1 Gy (Reg 2.5) , 6.2 Gy (Reg 5.0) and 6.0 Gy 

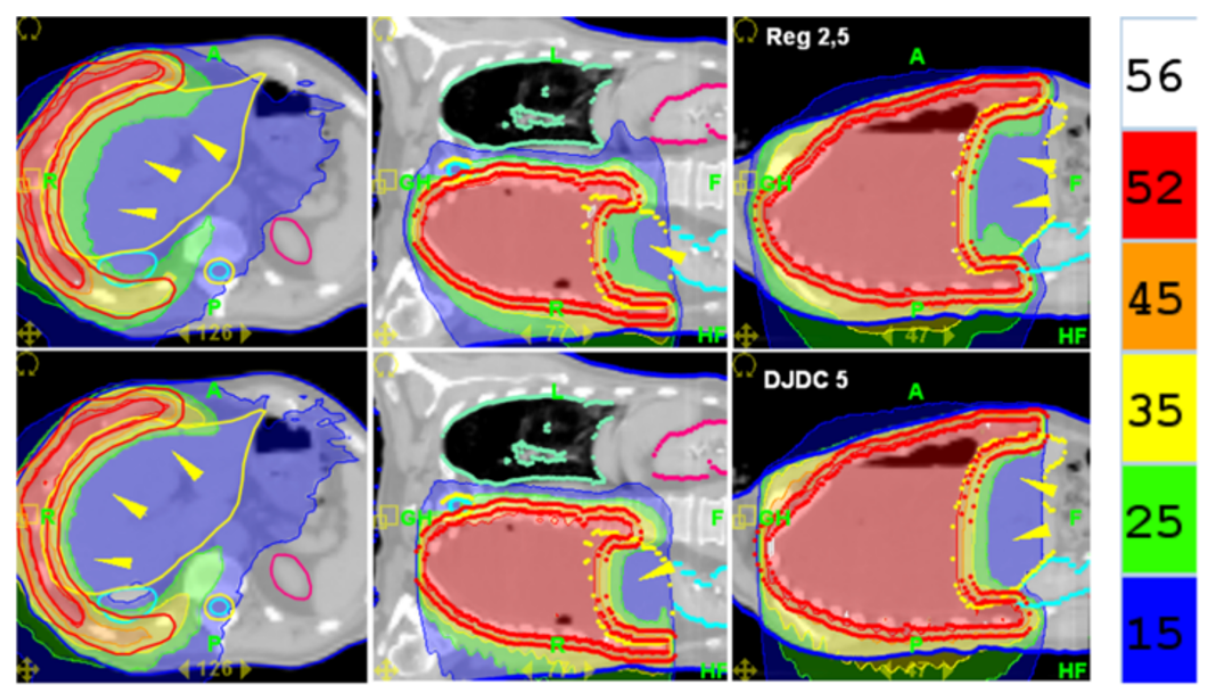

Figure 2 Hemithoracic Irradiation: Dose distributions for Reg 2.5 ("regular" delivery with a $2.5 \mathrm{~cm}$ field width; upper row) and DJDC 5.0 (Dynamic Jaw/Dynamic Couch delivery with a $5.0 \mathrm{~cm}$ maximum field width; lower row). Note the sparing of the liver in both modalities (arrowheads) and the reduced craniocaudal dose penumbra in DJDC plans.

(DJDC 5.0), respectively. Likewise, OAR sparing did not differ significantly in the WAI plans except for a higher bone exposure in Reg 5.0. Most notably, exposure of the liver and both kidneys remained on the same level.

For TMI, mean dose exposure of brain, lenses and lungs remained on a similar level in all delivery modes, while bladder, small bowel and stomach experienced a non-significant dose increase in Reg 5.0 and DJDC 5.0. Kidneys, parotid glands and thyroid gland received a significantly lower dose ( $\sim$ Gy) with Reg 2.5 compared to DJDC 5.0, but not to Reg 5.0.
The lungs, however, being the dose-limiting organs, received very similar mean doses of about 8 Gy in all modalities.

\section{Discussion}

This study is the first to show the characteristics of dynamic HT delivery for very large treatment volumes. In all three settings investigated we saw, as expected, a substantial reduction of up to $55 \%$ in treatment time when using $5 \mathrm{~cm}$ field width instead of $2.5 \mathrm{~cm}$. As a result, plans with Reg 5.0 showed an increased integral dose
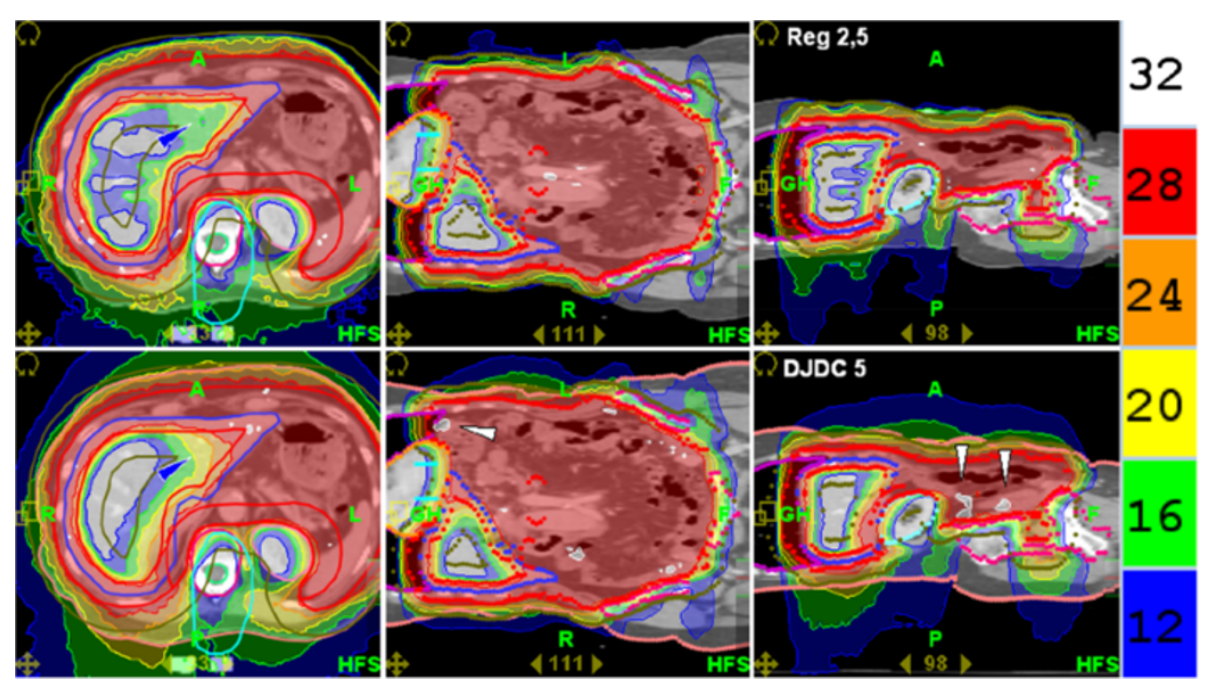

Figure 3 Whole Abdominal Irradiation: Dose distributions for Reg 2.5 ("regular" delivery with a $2.5 \mathrm{~cm}$ field width; upper row) and DJDC 5.0 (Dynamic Jaw/Dynamic Couch delivery with a $5.0 \mathrm{~cm}$ maximum field width; lower row). Note the slightly worse lateral dose penumbra in DJDC 5, for example in the left lobe of the liver (dark arrowhead) and the increased incidence of hotspots in DJCD (light arrowheads). 


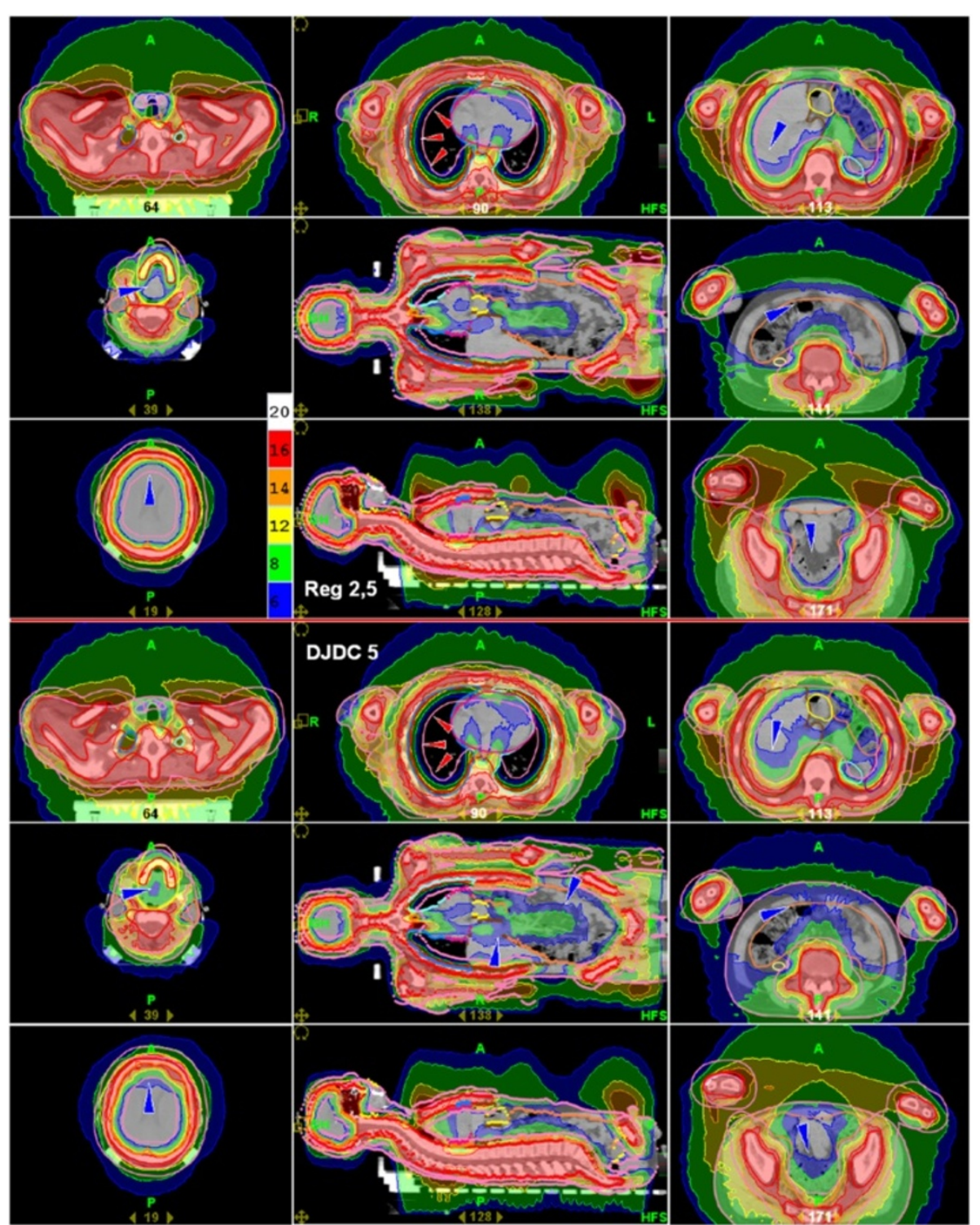

Figure 4 Total Marrow Irradiation: Dose distributions for Reg 2.5 ("regular" delivery with a $2.5 \mathrm{~cm}$ field width; upper half) and DJDC 5.0 (Dynamic Jaw/Dynamic Couch delivery with a $5.0 \mathrm{~cm}$ maximum field width; lower half). Note the equivalent lung sparing in both modalities (light arrowheads), which is accompanied by a more severe dose fall-off in the thoracic wall in DJDC 5.0. The dark arrowheads indicate areas of higher dose exposure in DJDC 5.0 that form the correlate for the non-significantly increased integral dose in DJDC 5.0.

and larger craniocaudal dose penumbra caused by a larger field width. This effect could be abolished with the use of DJDC delivery which produced fast treatment plans with a sharp craniocaudal dose-falloff. Aside from economic advantages based on a higher patient throughput, shorter treatments primarily increase patient comfort and treatment safety by lowering the risk of intrafractional movement. While treatment time reduction is attractive in every clinical setting, this feature is particularly important for the treatment for very large volumes: For a small target with 1.5 min treatment time, a $50 \%$ increase in speed is a small gain compared to the time needed for setup and image guidance. However, speeding up beam-on-time from $30 \mathrm{~min}$ to $15 \mathrm{~min}$ for large targets constitutes a substantial benefit.

\section{Hemithoracic irradiation}

For patients with Malignant Pleural Mesothelioma, trimodal treatment with extensive surgery including resection of the lung, the visceral and parietal pleura, the pericardium and the diaphragm (extrapleural pneumonectomy, EPP) followed by adjuvant radiotherapy and chemotherapy is a radical treatment approach [11]. The radiooncological target volume consists of the whole former pleural 

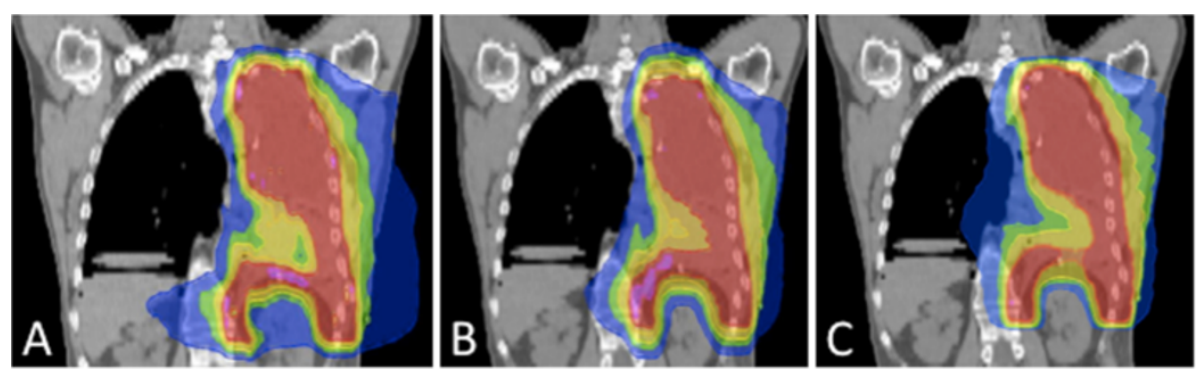

Figure 5 Hemithoracic Irradiation: DJDC 5.0 (C; Dynamic Jaw/Dynamic Couch delivery with a $5.0 \mathrm{~cm}$ maximum field width) plans resulted in a reduced dose penumbra compared to Reg 2.5 ( $\mathrm{A}$; ("regular" delivery with a $2.5 \mathrm{~cm}$ field width) and Reg 5.0 ("regular" delivery with a $5.0 \mathrm{~cm}$ field width).

cavity and is in close contact to critical structures such as the contralateral lung, the heart, the liver, the small bowel and the kidney. As pointed out by Allen et al. [12], patients after EPP are more susceptible to lung toxicity than patients without surgery. In a series of 13 patients, 6 developed fatal pneumonitis after fixed-beam IMRT treatment with a mean lung exposure of 15 Gy. HT shows dosimetric advantages over fixed-beam IMRT in terms of dose homogeneity and target coverage as well as OAR sparing. Mean dose to the contralateral lung can be lowered beyond 5 Gy with current HT with $2.5 \mathrm{~cm}$ field width [1]. The use of a bigger field width to spare treatment time has not proven clinically feasible because of a large dose penumbra to the neck and the upper abdomen, most notably the liver for right-sided mesothelioma (see Figure 5).

Yet, with DJDC technique, the present study could demonstrate similar dose distributions to "regular" HT. With DJDC 5.0, a drastic reduction of 55\% in treatment time could be realised. Considering a treatment schedule of 27 fractions, this would translate into a total reduction of beam-on-time of $2 \mathrm{~h} 36 \mathrm{~min}$.
Volumetric modulated arc therapy promises very fast IMRT treatments. In the case of 54 Gy hemithoracic irradiation, when comparing fixed-beam IMRT plans to RapidArc $^{\circledR}$ (RA) plans, Scorsetti et al. [13] found equivalent coverage and comparable OAR sparing, while treatment time (from loading of the data to the end of delivery, but excluding time for imaging and patient positioning) was significantly lower for RA (3.7 min vs. $13.4 \mathrm{~min}$ ). Direct comparison of different planning studies is prone to misinterpretations due to different CTV definitions and OAR contouring; however, compared to our DJDC data, Scorsetti et al. achieved a faster treatment with RA (average 5:10 min vs. 3:42 min, DJDC vs. RA) with comparable dose conformity and sparing of the contralateral lung and kidney. A comparison of liver exposure is impossible as our planning study included more patients with right-sided disease. Target coverage ( $\mathrm{TV}_{95 \%} 96,7 \%$ vs. 93.5 , DJDC vs. RA) and sparing of the ipsilateral kidney (mean dose 8.4 Gy vs. 12.9 Gy, DJDC vs. RA) were better in the DJDC plans.

When discussing the technical advances in hemithoracic irradiation after EPP, however, one has to take into
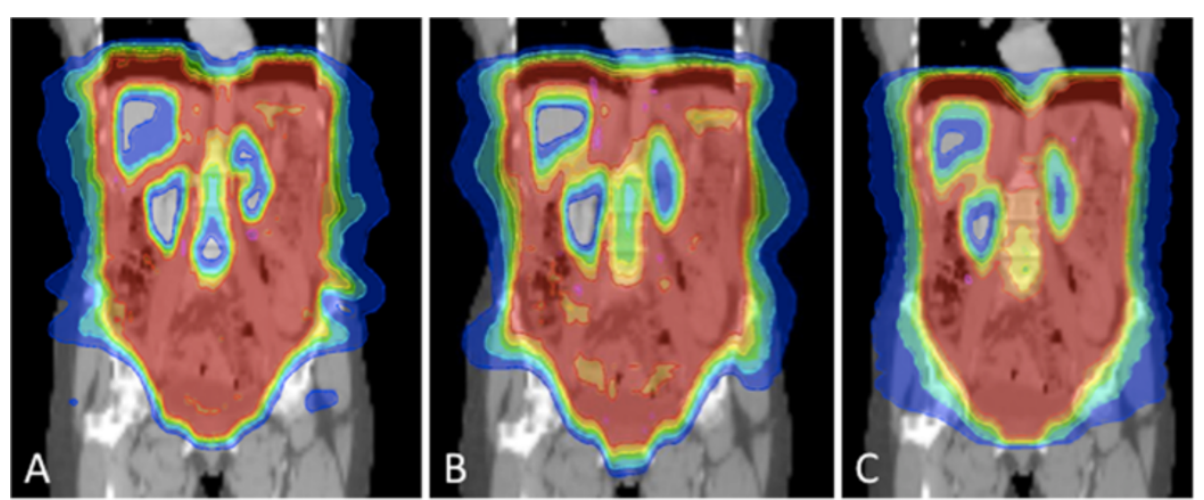

Figure 6 Whole Abdominal Irradiation: Vaginal sparing was better with DJDC 5.0 (C; Dynamic Jaw/Dynamic Couch delivery with a 5.0 $\mathrm{cm}$ maximum field width) compared to Reg 2.5 (A; ("regular" delivery with a $2.5 \mathrm{~cm}$ field width) and Reg 5.0 ("regular" delivery with a $5.0 \mathrm{~cm}$ field width) due to a smaller dose penumbra. 
account recent data of a randomised UK trial [14] comparing chemotherapy alone to trimodal treatment including EPP in 50 patients. While acute and late radiotherapy side effects in the EPP group were low with only two cases of grade 3 pneumonitis, 1-year overall survival was $52.2 \%$ in the EPP group compared to $73.1 \%$ in the no EPP group. Among the 16 patients that completed surgery, 4 perioperative deaths occurred. After adjustment for prognostic factors, the hazard ratio for overall survival between the EPP and no EPP group was 2.75. The authors conclude that EPP in a trimodal setting is associated with high morbidity and offers no benefit. Consequentially, EPP has been abandoned in many centers. Even so, the data presented in this study can be applied to other clinical situations such as lung cancer recurrence in the pleural cavity after pneumonectomy or soft tissue sarcoma of the pleural cavity as well.

\section{Whole abdominal irradiation}

Patients with advanced-stage ovarian cancer face a high risk of recurrence in the peritoneal cavity even after maximum cytoreductive surgery and adjuvant chemotherapy, while the risk of metastatic disease outside the abdomen is relatively low [15]. With a 5-year survival rate for patients with FIGO stage III ovarian cancer of 20-25\%, long-term prognosis is poor. The integration of Whole Abdominal Irradiation (WAI) into a multimodal consolidation concept has been evaluated with contradictory results, mainly because of high toxicity rates leading to delayed or incomplete radiotherapy [16]. With conventional radiotherapy techniques, delivery of adequate doses to the upper abdomen is impossible due to hepatic and renal toxicity [17]. The introduction of IMRT into clinical routine has opened new possibilities for a less toxic WAI as shown in a phase I trial [2] which might help to increase the locoregional control of locally advanced ovarian cancer. A phase I/II trial using HT is currently recruiting in our department [18].

With Reg 2.5, one WAI treatment fraction of 1.5 Gy takes about 18 minutes. Should WAI be integrated in routine treatment regimes for ovarian cancer, treatment acceleration will become an important issue. Using Reg 5.0 caused a significant drop in target coverage due to the larger field width. DJDC 5.0 plans also showed a significantly, but not as pronounced, loss in coverage. The dynamic jaw component could partly compensate the negative effect of the larger field width. In contrast, DJDC could not counterbalance the higher liver exposure caused by $5.0 \mathrm{~cm}$ jaw opening, probably because the gradient to the liver lies more in diagonal direction than in craniocaudal direction. Yet, liver exposure was only increased by about $6 \%$ which we believe is not clinically relevant.
Mahantshetty et al. [19] compared WAI with fixedbeam IMRT to RA with three arcs and two isocenters, using $6 \mathrm{MV}$ and $15 \mathrm{MV}$ for both techniques and prescribing 25 Gy to the whole abdomen and a simultaneous integrated boost of $45 \mathrm{~Gy}$ to the pelvis. They found comparable target coverage and slightly improved homogeneity for RA. In terms of OAR sparing, $15 \mathrm{MV}$ plans were superior to lower energy plans; 15 MV IMRT and 15 MV RA showed a basic equivalence. Average treatment time was significantly lower for RA (4:8 min for $6 \mathrm{MV}$ and 15 MV RA vs. 18:0 min for 6 MV IMRT and 17:4 min for $15 \mathrm{MV}$ IMRT). As dose prescription differs strongly from our plans, a reliable comparison to our data is difficult. When scaling down our PTV dose to 25 Gy, DJDC would yield a superior liver sparing (mean dose 19.8 Gy vs. 22.4 Gy, DJDC vs. RA).

\section{Total marrow irradiation}

As part of the conditioning regimen before autologuous stem cell transplantation, Total Body Irradiation (TBI) plays a role in the treatment of a broad range of hematological malignancies such as acute lymphoblastic leukemia [20]. However, in the conditioning regimens of other diseases such as Multiple Myeloma, TBI has been abandoned due to extensive toxicity, particularly mucositis, dependence on i.v. nutrition and hospitalisation time $[21,22]$. The standard TBI technique uses large ap/pa and lateral fields while blocking the lungs and an electron boost to the thoracic wall and is associated with substantial toxicity. While restriction of the target volume to the bone marrow with conventional techniques is still associated with substantial toxicity [23], modern IMRT techniques promise a further reduction of OAR exposure [24]. Technical and clinical feasibility of Total Marrow Irradiation (TMI) using HT have been demonstrated by Wong et al. in 2006 [25]. In a phase I dose escalation trial, TMI was investigated as part of a tandem autologuous stem cell transplantation regimen for patients with Multiple Myeloma. Dose-limiting toxicity was determined at 18 Gy. Up to a total dose of 16 Gy, TMI was tolerated well with a low incidence of grade 3 nonhematological toxicities [26]. One major drawback of advanced TMI techniques are, however, the long treatment times required for highly conformal dose delivery. For state-of-the art HT, treatment times of 20-40 min are reported, depending on the chosen field width [27].

For technical reasons, the maximum target length for HT is limited to $160 \mathrm{~cm}$. Like most other groups using HT for TMI, we would address this problem by treating the lower extremities with ap/pa fields on a linear accelerator.

In the present study, Reg 2.5, Reg 5.0 and DJDC 5.0 could deliver the same dose to the target. The lungs as 
the dose-limiting organs caused a dose fall-off to about 12 Gy particularly in Reg 5.0 and DJDC 5.0 delivery, which is probably due to the larger maximum field width. This phenomenon can also be seen in the DVHs published by Han et al. [27]. Regarding a prescribed dose of $18 \mathrm{~Gy}$, this appears to be an unacceptable underdosage at first glance. However, this is the dose level the patient would have received with conventional TBI to the whole target. Thus, with both modalities, $99 \%$ of the PTV received the conventional TBI dose or more, and $76 \%$ received 17.1 Gy or more.

Some OAR were exposed to a significantly higher mean dose with DJDC 5.0 compared to Reg 2.5, in particular the liver, the kidneys, the parotid glans and the thyroid gland. As the Reg 5.0 plans showed no significant dose increase in those organs, this might be an effect of the dynamic component. Yet, OAR exposure was raised by approximately 1 Gy which is probably not clinically relevant.

Current HT is often criticised because of long treatment times and challenged by volumetric arc modulated techniques that promise faster treatments. Very recently, Han et al. [27] showed in a plan comparison study for TMI a reduction of average beam-on time from 1122 sec to $628 \mathrm{sec}$ when using RA instead of HT with $5 \mathrm{~cm}$ field width. In addition, mean OAR dose could be lowered significantly in some organs such as the brain, the right kidney, the optic nerves and the thyroid gland. However, mean dose to the intestines as well as D10 (dose covering 10\% of the volume) for the intestines, both lungs and the stomach were significantly higher in RA plans. On high dose levels (D80), no difference in lung exposure could be detected. In addition to beamon-time, time for isocenter setup is needed for each arc field in RA technique. Thus, the authors estimate that total treatment time for RA and HT would be comparable. Our results with an average treatment time of 1105 sec for TMI are in line with their data.

One feature of DJDC technique is its ability to diminish the craniocaudal dose penumbra and consecutively the integral dose as demonstrated for nasopharyngeal cancer [28]. Contrary to expectations, in the present study, a significantly lower integral dose with DJDC as compared to Reg 5.0 could only be demonstrated for WAI. In all other settings, integral dose did not differ significantly between Reg 2.5, Reg 5.0 and DJDC 5.0, which is probably due to crucial differences in PTV shape: For nasopharyngeal cancer, the relation of longitudinal to lateral extension is much smaller than for the large volumens investigated in this study, giving the size of the longitudinal penumbra a bigger influence. In addition, the main dose gradient in nasopharyngeal cancer treatment (i.e. towards the myelon) is situated more or less on the same coronal plane. In contrast, the gradients in Hemithoracic Irradiation, WAI in TMI vary in location and cross the coronal planes, e.g. along the lower rim of the liver. In such a setting, the bigger maximum field width in DJDC causes a bigger lateral dose penumbra and outweighs the gain achieved by the reduced craniocaudal penumbra.

\section{Conclusions}

The HT treatment of large volumes such as the hemithorax, the abdominal cavity or the complete bone marrow can be sped up substantially by DJDC, while maintaining excellent target coverage, OAR sparing and sharp dose gradients above and below the target. Especially in the case of targets with very large craniocaudal extensions such as Total Marrow Irradiation, it constitutes a convenient and safe treatment modality. However, for the time being, dynamic HT is still under development and not available for clinical routine yet.

\section{Abbreviations}

DJDC: Dynamic jaw/dynamic couch; HT: Helical tomotherapy; IMF: Intensity modulation factor; IMRT: Intensity-modulated radiotherapy; OAR: Organ at risk; MLC: Multi-leaf-collimator; TMI: Total marrow irradiation; WAl: Whole abdominal irradiation; $D_{1 / 99}$ : Dose to $1 \% / 99 \%$ of the target volume; $\mathrm{Cl}_{95}$ : Conformity index total volume covered by the $95 \%$ isodose divided by the volume of the PTV covered by the 95\% isodose; UI: Uniformity index dose covering $5 \%$ of the PTV divided by the dose covering $95 \%$ of the PTV; $\mathrm{TV}_{95 \%}$ : Volume covered by $95 \%$ of the prescribed dose divided by the PTV volume; EPP: Extrapleural pneumonectomy; RA: RapidArc ${ }^{\circledR}$.

\section{Competing interests}

The authors have no competing interests to declare.

\section{Authors' contributions}

SK was responsible for drafting the manuscript. SB did the treatment planning and plan evaluation. KS and SL were responsible for data and software management. KH contributed to the revision of the manuscript. JD as head of the Department of Radiation Oncology has given final approval to the article. FS as head of the Tomotherapy research group at the Department of Radiation Oncology was responsible for conception and design of the study, treatment planning and revision of the manuscript. All authors read and approved the final manuscript.

\section{Acknowledgements}

This work has been supported by the Faculty of Medicine, University of Heidelberg, Germany. The department of Radiation Oncology, University Hospital of Heidelberg has a research cooperation with Tomotherapy Inc.

\section{Author details}

'Department of Radiation Oncology, University Hospital Heidelberg, INF 400, 69120, Heidelberg, Germany. ${ }^{2}$ Department of Therapeutic Radiology, University of Minnesota, 420 Delaware St. SE, Mayo Mail Code 494, Minneapolis, MN 55455, USA.

Received: 3 May 2012 Accepted: 5 November 2012

Published: 12 November 2012

\section{References}

1. Sterzing F, Sroka-Perez G, Schubert K, Munter MW, Thieke C, Huber P, Debus J, Herfarth KK: Evaluating target coverage and normal tissue sparing in the adjuvant radiotherapy of malignant pleural mesothelioma: helical tomotherapy compared with step-and-shoot IMRT. Radiother Oncol 2008, 86:251-257.

2. Rochet $N$, Jensen AD, Sterzing F, Munter MW, Eichbaum MH, Schneeweiss A, Sohn C, Debus J, Harms W: Adjuvant whole abdominal intensity modulated radiotherapy (IMRT) for high risk stage FIGO III patients with 
ovarian cancer (OVAR-IMRT-01) - Pilot trial of a phase I/II study: study protocol. BMC Cancer 2007, 7:227.

3. Mackie TR, Holmes T, Swerdloff S, Reckwerdt P, Deasy JO, Yang J, Paliwal B, Kinsella T: Tomotherapy: a new concept for the delivery of dynamic conformal radiotherapy. Med Phys 1993, 20:1709-1719.

4. Welsh JS, Patel RR, Ritter MA, Harari PM, Mackie TR, Mehta MP: Helical tomotherapy: an innovative technology and approach to radiation therapy. Technol Cancer Res Treat 2002, 1:311-316.

5. Shepard DM, Olivera GH, Reckwerdt PJ, Mackie TR: Iterative approaches to dose optimization in tomotherapy. Phys Med Biol 2000, 45:69-90.

6. Parker W, Brodeur M, Roberge D, Freeman C: Standard and nonstandard craniospinal radiotherapy using helical TomoTherapy. Int I Radiat Oncol Biol Phys 2010, 77:926-931.

7. Otto K: Volumetric modulated arc therapy: IMRT in a single gantry arc. Med Phys 2008, 35:310-317.

8. Bortfeld T, Webb S: Single-Arc IMRT? Phys Med Biol 2009, 54:N9-20

9. Yang JN, Mackie TR, Reckwerdt P, Deasy JO, Thomadsen BR: An investigation of tomotherapy beam delivery. Med Phys 1997, 24:425-436

10. Chen Y, Chen Q, Chen M, Lu W: Dynamic tomotherapy delivery. Med Phys 2011, 38:3013-3024.

11. Sugarbaker DJ, Flores RM, Jaklitsch MT, Richards WG, Strauss GM, Corson JM, DeCamp MM Jr, Swanson SJ, Bueno R, Lukanich JM, et al: Resection margins, extrapleural nodal status, and cell type determine postoperative long-term survival in trimodality therapy of malignant pleural mesothelioma: results in 183 patients. J Thorac Cardiovasc Surg 1999, 117:54-63. discussion 63-55.

12. Allen AM, Czerminska M, Janne PA, Sugarbaker DJ, Bueno R, Harris JR, Court L, Baldini EH: Fatal pneumonitis associated with intensity-modulated radiation therapy for mesothelioma. Int J Radiat Oncol Biol Phys 2006, 65:640-645.

13. Scorsetti M, Bignardi M, Clivio A, Cozzi L, Fogliata A, Lattuada P, Mancosu P, Navarria P, Nicolini G, Urso G, et al: Volumetric modulation arc radiotherapy compared with static gantry intensity-modulated radiotherapy for malignant pleural mesothelioma tumor: a feasibility study. Int J Radiat Oncol Biol Phys 2010, 77:942-949.

14. Treasure T, Lang-Lazdunski L, Waller D, Bliss JM, Tan C, Entwisle J, Snee M, O'Brien M, Thomas G, Senan S, et al: Extra-pleural pneumonectomy versus no extra-pleural pneumonectomy for patients with malignant pleural mesothelioma: clinical outcomes of the Mesothelioma and Radical Surgery (MARS) randomised feasibility study. Lancet Oncol 2011, 12:763-772.

15. Ozols RF, Bundy BN, Greer BE, Fowler JM, Clarke-Pearson D, Burger RA, Mannel RS, DeGeest K, Hartenbach EM, Baergen R: Phase III trial of carboplatin and paclitaxel compared with cisplatin and paclitaxel in patients with optimally resected stage III ovarian cancer: a Gynecologic Oncology Group study. J Clin Oncol 2003, 21:3194-3200.

16. Thomas GM: Is there a role for consolidation or salvage radiotherapy after chemotherapy in advanced epithelial ovarian cancer? Gynecol Oncol 1993, 51:97-103.

17. Dembo AJ: Abdominopelvic radiotherapy in ovarian cancer. A 10-year experience. Cancer 1985, 55:2285-2290.

18. Rochet N, Kieser M, Sterzing F, Krause S, Lindel K, Harms W, Eichbaum MH, Schneeweiss A, Sohn C, Debus J: Phase II study evaluating consolidation whole abdominal intensity-modulated radiotherapy (IMRT) in patients with advanced ovarian cancer stage FIGO III-the OVAR-IMRT-02 Study. BMC Cancer 2011, 11:41.

19. Mahantshetty U, Jamema S, Engineer R, Deshpande D, Sarin R, Fogliata A, Nicolini G, Clivio A, Vanetti E, Shrivastava S, Cozzi L: Whole abdomen radiation therapy in ovarian cancers: a comparison between fixed beam and volumetric arc based intensity modulation. Radiat Oncol 2010, 5:106

20. Hill-Kayser CE, Plastaras JP, Tochner Z, Glatstein E: TBI during BM and SCT: review of the past, discussion of the present and consideration of future directions. Bone Marrow Transplant 2011, 46:475-484.

21. Goldschmidt $H$, Hegenbart U, Wallmeier M, Hohaus S, Engenhart R, Wannenmacher M, Haas R: Peripheral blood progenitor cell transplantation in multiple myeloma following high-dose melphalanbased therapy. Recent Results Cancer Res 1998, 144:27-35.

22. Moreau P, Facon T, Attal M, Hulin C, Michallet M, Maloisel F, Sotto J, Guilhot F, Marit G, Doyen C, et al: Comparison of $200 \mathrm{mg} / \mathrm{m}(2)$ melphalan and $8 \mathrm{~Gy}$ total body irradiation plus $140 \mathrm{mg} / \mathrm{m}(2)$ melphalan as conditioning regimens for peripheral blood stem cell transplantation in patients with newly diagnosed multiple myeloma: final analysis of the Intergroupe Francophone du Myelome 9502 randomized trial. Blood 2002, 99:731-735.

23. Zaucha RE, Buckner DC, Barnett T, Holmberg LA, Gooley T, Hooper HA, Maloney DG, Appelbaum F, Bensinger WI: Modified total body irradiation as a planned second high-dose therapy with stem cell infusion for patients with bone-based malignancies. Int I Radiat Oncol Biol Phys 2006, 64:227-234

24. Schultheiss TE, Wong J, Liu A, Olivera G, Somlo G: Image-guided total marrow and total lymphatic irradiation using helical tomotherapy. Int J Radiat Oncol Biol Phys 2007, 67:1259-1267.

25. Wong JY, Liu A, Schultheiss T, Popplewell L, Stein A, Rosenthal J, Essensten M, Forman S, Somlo G: Targeted total marrow irradiation using threedimensional image-guided tomographic intensity-modulated radiation therapy: an alternative to standard total body irradiation. Biol Blood Marrow Transplant 2006, 12:306-315.

26. Somlo G, Spielberger R, Frankel P, Karanes C, Krishnan A, Parker $P$, Popplewell L, Sahebi F, Kogut N, Snyder D, et al: Total marrow irradiation: a new ablative regimen as part of tandem autologous stem cell transplantation for patients with multiple myeloma. Clin Cancer Res 2011, 17:174-182.

27. Han C, Schultheisss TE, Wong JY: Dosimetric study of volumetric modulated arc therapy fields for total marrow irradiation. Radiother Oncol 2012, 102:315-320

28. Sterzing F, Uhl M, Hauswald H, Schubert K, Sroka-Perez G, Chen Y, Lu W, Mackie R, Debus J, Herfarth K, Oliveira G: Dynamic jaws and dynamic couch in helical tomotherapy. Int J Radiat Oncol Biol Phys 2010, 76:1266-1273

doi:10.1186/1748-717X-7-191

Cite this article as: Sonja et al:: Accelerated large volume irradiation with dynamic Jaw/Dynamic Couch Helical Tomotherapy. Radiation Oncology 2012 7:191.

\section{Submit your next manuscript to BioMed Central and take full advantage of:}

- Convenient online submission

- Thorough peer review

- No space constraints or color figure charges

- Immediate publication on acceptance

- Inclusion in PubMed, CAS, Scopus and Google Scholar

- Research which is freely available for redistribution 